\begin{tabular}{|c|c|}
\hline Title & $\begin{array}{l}\text { Specific A bsorption Rates of A natomically Realistic Human Models Exposed to RF Electromagnetic Fields From } \\
\text { Mobile PhonesU sed in Elevators }\end{array}$ \\
\hline Author(s) & Simba, Ally Y.; Hikage, Takashi; W atanabe, Soichi; Nojima, Toshio \\
\hline Citation & $\begin{array}{l}\text { IEEE Transactions on Microwave Theory and Techniques, 57(5-1), 1250-1259 } \\
\text { https://doi.org/10.1109/1 MTT.2009.2017354 }\end{array}$ \\
\hline Issue Date & $2009-05$ \\
\hline Doc URL & http:/hdl. handle.net/2115/38548 \\
\hline Rights & $\begin{array}{l}\text { (c) 2009 IEEE. Personal use of this material is permitted. However, permission to reprint/republish this material for } \\
\text { advertising or promotional purposes or for creating new collective works for resale or redistribution to servers or lists, } \\
\text { or to reuse any copyrighted component of this work in other works must be obtained from the IEEE. }\end{array}$ \\
\hline Type & article \\
\hline File Information & 57-5-1_p1250-1259.pdf \\
\hline
\end{tabular}

Instructions for use 


\title{
Specific Absorption Rates of Anatomically Realistic Human Models Exposed to RF Electromagnetic Fields From Mobile Phones Used in Elevators
}

\author{
Ally Y. Simba, Member, IEEE, Takashi Hikage, Member, IEEE, \\ Soichi Watanabe, Member, IEEE, and Toshio Nojima, Member, IEEE
}

\begin{abstract}
This paper presents a detailed numerical investigation to determine whether or not an increased specific absorption rate (SAR) in an adult using a mobile phone inside an elevator due to the multireflections of electromagnetic fields from the walls exceed the RF-exposure guidelines. A fully realistic heterogeneous human body model and an actual elevator size were employed. The nonuniform mesh finite-difference time-domain technique and a supercomputer were employed to obtain the SAR and other important parameters. The mobile phone was modeled as a $\lambda / 2$ dipole antenna placed at a distance of $16 \mathbf{~ m m}$ from the head. For computations, operating frequencies of 900,1500 , and $2000 \mathrm{MHz}$ with transmitting power of $250 \mathrm{~mW}$ were used. Computed results show that the peak spatial-average 10 -g SAR depends on the position of the passenger and the antenna against the elevator walls. We observed a substantial increase in the whole-body average SAR and peak 10-g SAR values of the passenger in the elevator over their respective free-space values. However, the maximum values obtained do meet the basic restrictions described in the international RF safety guidelines. For example, the maximum values of the whole-body average and peak spatial-average SAR were $4.4 \%$ and $78 \%$ of the international RF safety guideline, respectively.
\end{abstract}

Index Terms-Finite-difference time-domain (FDTD) technique, multireflections, realistic human model, specific absorption rate (SAR).

\section{INTRODUCTION}

W ITH THE rapid increase in the use of mobile phones in enclosed environments such as trains, cars, and elevators, public anxiety over the possibility of RF exposures in these environments exceeding the basic restrictions of the International Commission on Non-Ionizing Radiation Protection (ICNIRP) [1] and IEEE guidelines [2] has been growing. It should be noted that according to the guidelines mentioned above, the only scientifically established adverse health effect of RF energy in the human body is thermal, and its safety limits are given based on specific absorption rates (SARs). Discussions of the nonthermal effects of RF exposure in the human body and the

Manuscript received November 16, 2008; revised November 16, 2008. First published April 14, 2009; current version published May 06, 2009.

A. Y. Simba and S. Watanabe are with the National Institute of Information and Communications Technology, Tokyo 184-8795, Japan (e-mail: simba@ nict.go.jp; wata@nict.go.jp).

T. Hikage and T. Nojima are with the Graduate School of Information Science and Technology, Hokkaido University, Sapporo 060-0814, Japan (e-mail: hikage@wtemc.ist.hokudai.ac.jp; nojima@wtemc.ist.hokudai.ac.jp).

Color versions of one or more of the figures in this paper are available online at http://ieeexplore.ieee.org.

Digital Object Identifier 10.1109/TMTT.2009.2017354 interference with implantable medical devices are beyond the scope of this study.

The public anxiety over the use of mobile phones in enclosed environments has risen partly because of some reports in academic journals suggesting that electromagnetic field (EMF) levels in these environments exceed the safety levels during mobile phone use [3]-[5]. Furthermore, the appearance of these reports in well-known international mainstream media such as the British Broadcasting Corporation (BBC) ${ }^{1}$ and others has probably fueled these concerns. On the other hand, there have been several attempts by researcher who do not agree with these reports to allay these fears. Several papers have been published on this concept to date [6]-[10].

After a thorough review of the studies on this subject, we have thus far found very little information, especially on the localized peak spatial-averaged 10-g SAR in a realistic human model in actual enclosed environments. This is by far the most important parameter in reaching any conclusion regarding the thermal effect of RF exposure. Some researchers have investigated this effect [11]-[13], but only on a passenger's head, while ignoring the rest of the body. The body in an enclosed environment like an elevator would also absorb electromagnetic energy. Furthermore, the head-and-metallic wall configuration does not represent a realistic case of a passenger in an elevator or other enclosed environment, especially when multireflections of the EMF from the walls are to be considered.

The lack of spatial SAR information may be partly due to the large-scale computer resources required to perform numerical investigations of a realistic human model in an actual elevator. Recently, a numerical investigation of this problem using a finite-difference time-domain (FDTD) technique was reported [14]; however, the report gives limited information of only the whole-body average SAR. Considering the above factors, we have previously presented preliminary investigations of this study [15].

The nonuniform mesh FDTD technique [16], which minimizes computer resources by a factor of almost five, and a supercomputer were used to perform detailed calculations of the SAR in an anatomically realistic human model inside an actual elevator at the cellular phone operating frequencies used in Japan (i.e., 900, 1500, and $2000 \mathrm{MHz}$ ). Specifically, we address two issues, which are: 1) whether or not the safety guidelines are exceeded in the elevator, by comparing maximum values obtained

${ }^{1}$ BBC News, Health, "Trains trap mobile phone radiation." [Online]. Available: http://news.bbc.co.uk/2/hi/health/1961484.stm 


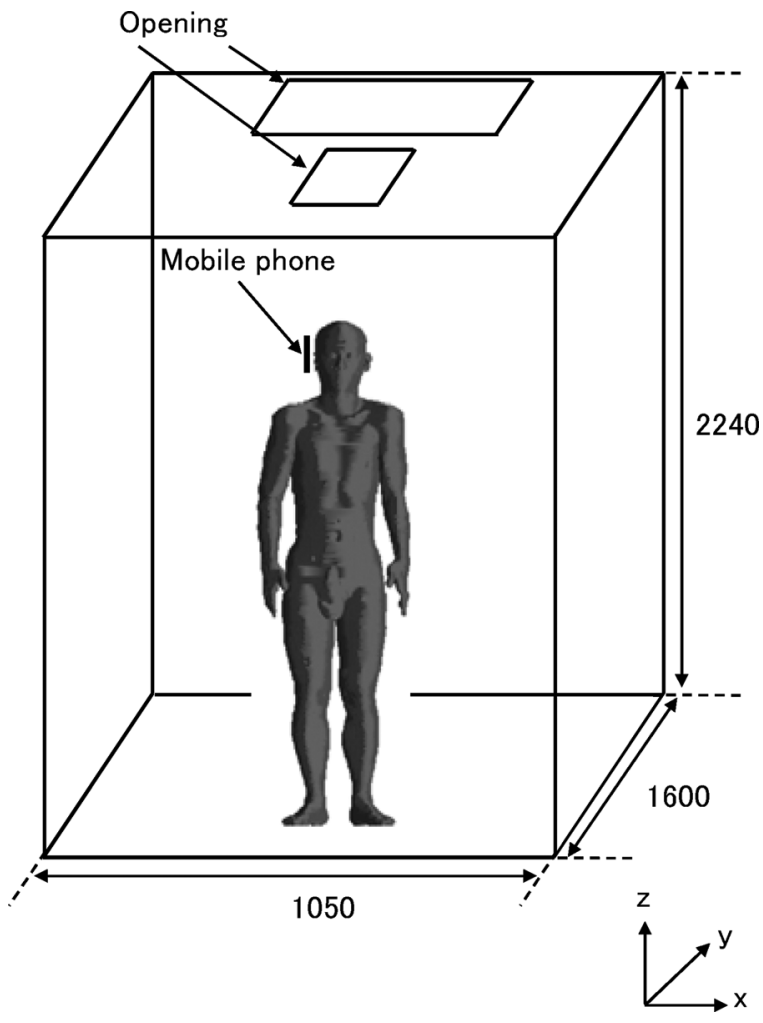

Fig. 1. Elevator with one mobile phone user (units: millimeters).

with the basic restrictions and 2) to provide an understanding of the SAR characteristics of the mobile phone user in such environments under worst case conditions.

The investigations in this paper will include the effect of the user's position inside the elevator and the influence of elevator's structure, such as its walls and openings. The "worst case" considered in this paper refers to conditions that include: 1) a halfwavelength dipole antenna, placed near the head (no hand model was used to hold the antenna like in a real-life mobile phone use); 2) a vertically oriented dipole antenna, parallel to the sidewalls of the elevator for maximum reflection; 3 ) an elevator with metallic walls; and 4) a constant maximum transmitting power of $250 \mathrm{~mW}$.

\section{Models of the Elevator AND PASSEnger}

The structure of the elevator is shown in Fig. 1. The dimensions of the elevator used in this study were taken from an actual elevator. Two small openings $(300 \mathrm{~mm} \times 600 \mathrm{~mm}$ and $300 \mathrm{~mm}$ $\times 300 \mathrm{~mm}$ ) were introduced on the roof. Although their original purpose may be to provide ventilation and to be used for maintenance, they can also be considered as the main propagation route for the radio waves into the elevator [17].

The elevator being investigated has a maximum capacity of nine passengers. However, in this paper, only the simple case of one passenger using a mobile phone was considered. In this condition, no power would be absorbed or blocked by other passengers. Therefore, the effect of multireflections from the walls was at a maximum and all power transmitted by the phone was absorbed by the single passenger.

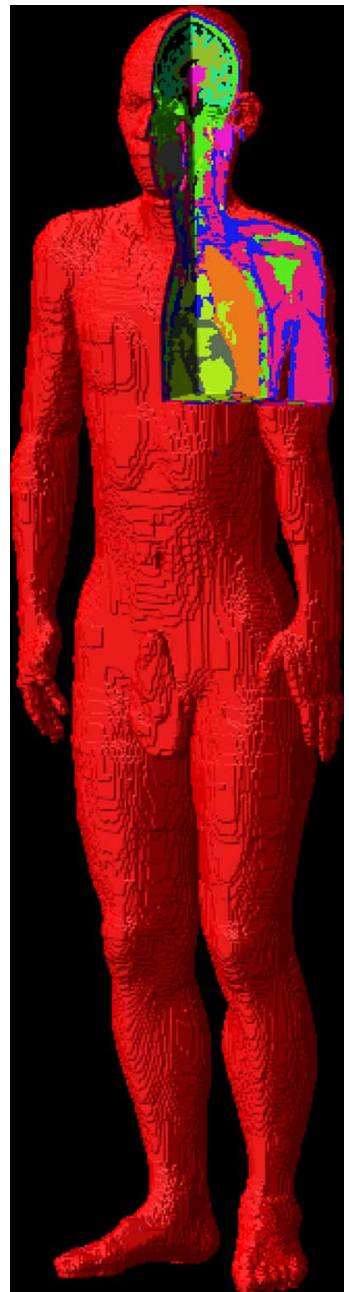

Fig. 2. Realistic Japanese adult male model used in the analysis.

A realistic high-resolution model of the Japanese adult male of average weight and height, shown in Fig. 2, is used to represent the passenger [18]. It consists of voxels of $2 \times 2 \times 2 \mathrm{~mm}^{3}$, manually segmented in to 51 different tissues and organs. The model is $172.8-\mathrm{mm}$ tall and weighed $65.0 \mathrm{~kg}$. Further details on the procedure of the development of the model are described in [18].

We also used an adult Japanese female model for comparative purposes. The female model, whose height and weight are $160 \mathrm{~mm}$ and $53.0 \mathrm{~kg}$, respectively, was developed using the same procedures as those employed to develop the male model, and has the same $2 \times 2 \times 2 \mathrm{~mm}^{3}$ voxel resolution [18].

The electrical properties used for the human models were derived from [19]-[22] and other sources described in these works.

A half-wavelength thin-wire dipole antenna placed vertically in the right side of the head at a distance $16 \mathrm{~mm}$ away and without a hand was used to represent a mobile phone. It has been reported elsewhere that the highest SAR of the head is obtained when no hand is used to hold the handset [23] and a vertically orientated half-wavelength dipole antenna [24], [25] is used.

During calculations, the models were grounded to the elevator floor through the feet. 


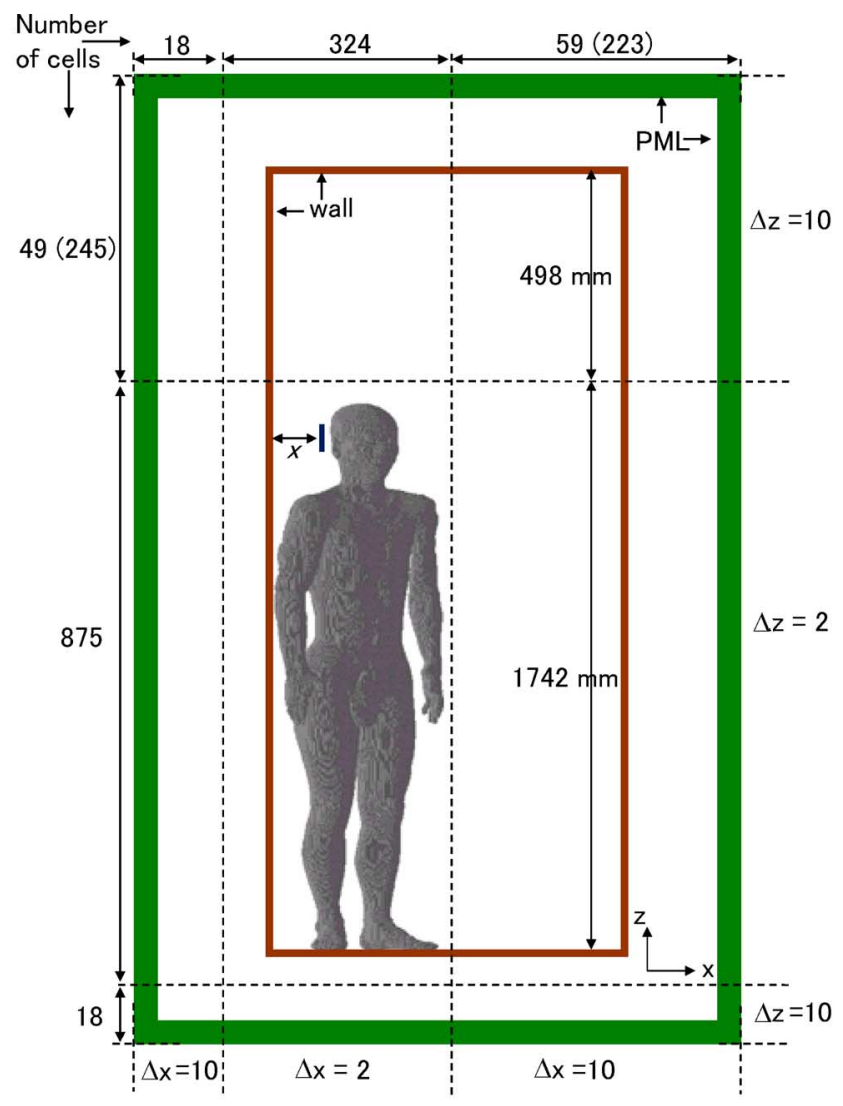

(a)

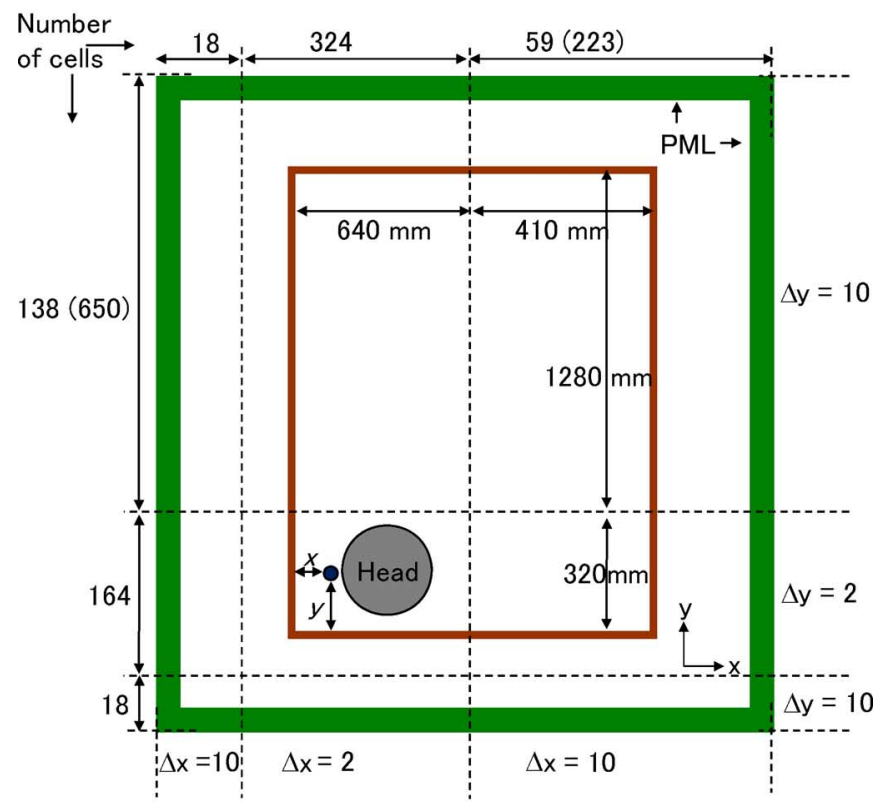

(b)

Fig. 3. Typical nonuniform mesh FDTD problem space of an elevator with a single passenger. $\Delta x, \Delta y$, and $\Delta z$ are the cell sizes used. The numbers of cells required are shown on the left and top of the problem space. The passenger position in relation to the elevator walls in the $x$ - and $y$-directions is indicated by $x$ and $y$. (a) $x z$-plane (front view). (b) $y z$-plane (top view).

\section{Method of CALCUlations}

The nonuniform mesh FDTD technique was used in this investigation to minimize computer resources. This technique,

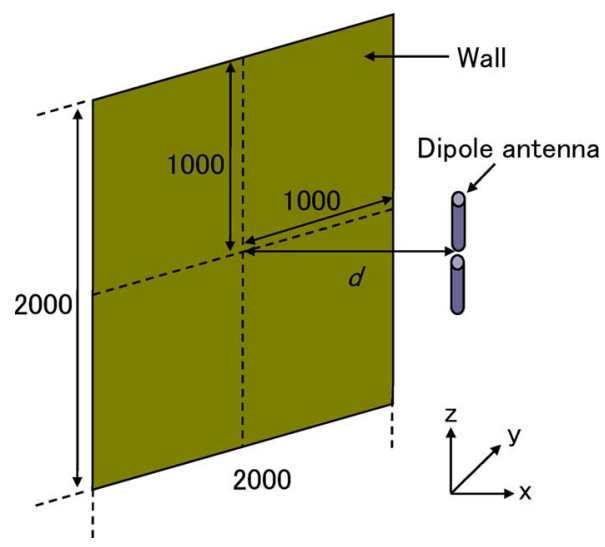

Fig. 4. Configuration of the dipole antenna close to a metallic wall (units: millimeters).

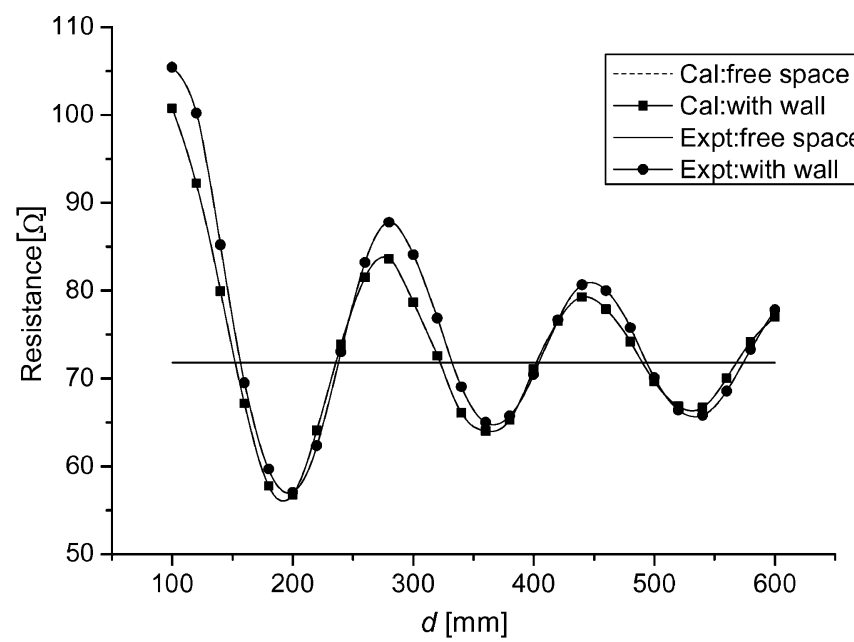

(a)

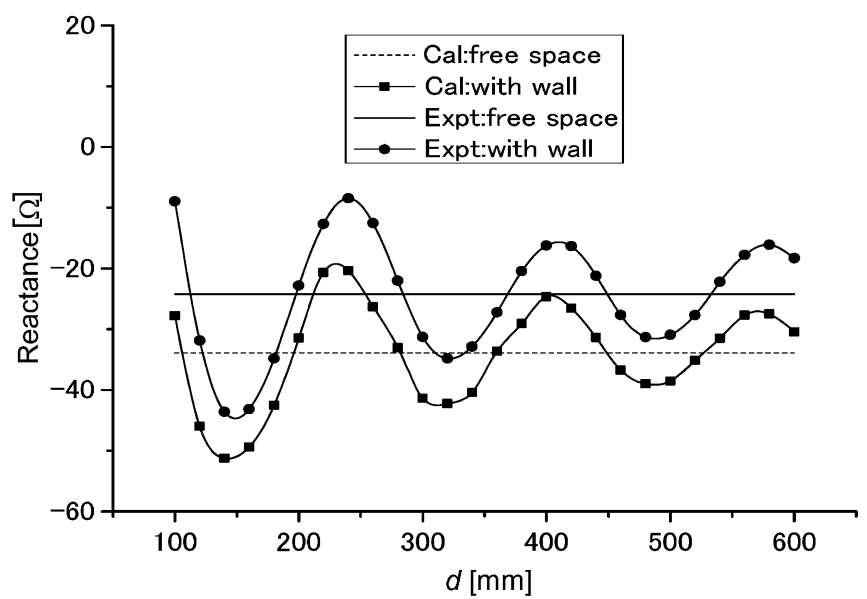

(b)

Fig. 5. Measured and calculated results of the input impedance of the halfwavelength dipole antenna as a function of the distance from the metallic wall, as shown in Fig. 4. (a) Real part of the input impedance. (b) Imaginary part of the input impedance.

which is based on Maxwell's curl equations represented by the midpoint method and Yee-cell notation, utilizes either a linear or quadratic interpolation at the coarse-fine cell boundary to reduce reflection errors [16]. 
TABLE I

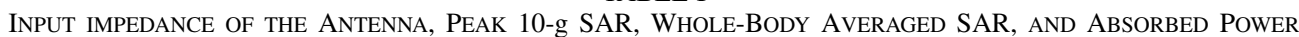
When a Half-WaVelength Dipole ANTENNa IS TRANSMitTing $250 \mathrm{~mW}$ at 900, 1500, and $2000 \mathrm{MHz}$

\begin{tabular}{ccccc}
\hline Frequency [MHz] & $\begin{array}{c}\text { Input Impedance } \\
{[\Omega]}\end{array}$ & $\begin{array}{c}\text { Peak 10-g SAR } \\
{[\mathrm{W} / \mathrm{kg}]}\end{array}$ & $\begin{array}{c}\text { Whole-body average } \\
\text { SAR }[\mathrm{mW} / \mathrm{kg}]\end{array}$ & $\begin{array}{c}\text { Absorbed Power } \\
{[\mathrm{mW}]}\end{array}$ \\
\hline 900 & $64+\mathrm{j} 67$ & 0.90 & 2.06 & 143 \\
1500 & $85+\mathrm{j} 109$ & 0.94 & 1.46 & 101 \\
2000 & $97+\mathrm{j} 80$ & 1.13 & 1.13 & 78 \\
\hline \hline
\end{tabular}

Throughout this study, fine-size cells of $\Delta x=\Delta y=\Delta z=$ $2 \mathrm{~mm}$ were used to model the regions around the dipole antenna and human model. Other areas of the FDTD problem space were modeled with coarse-size cells of $10 \mathrm{~mm}$ in all directions. The coarse-size cell must be $\leq \lambda / 15$ [16], where $\lambda$ is the free-space wavelength. A coarse-size cell of $10 \mathrm{~mm}$ used in this study satisfy this condition for up to $2000 \mathrm{MHz}$. Note that, in this study, the fine-size cell was chosen to accommodate the human model, whose voxels have $2 \times 2 \times 2 \mathrm{~mm}^{3}$ resolution. The time step in the nonuniform mesh FDTD technique is always set by taking into consideration the smallest fine-cell size; in our case, $2 \mathrm{~mm}$, and the Courant condition. The time steps used in the simulation were $3.81,3.79$, and 3.79 ps for 900,1500 and $2000 \mathrm{MHz}$, respectively.

Beranger's perfect matched layer (PML) [26] having eight layers and $M=4$ are placed at all boundaries of the FDTD problem space to absorb the radiated outgoing waves. Elevator walls, assumed to be of a metallic material, were modeled as a perfect electric conductor (PEC) by setting all $E$-fields to be equal to zero at the wall's surface.

The nonuniform mesh FDTD problem space of the elevator with one passenger is shown in Fig. 3, which indicates the cell size and number of cells used. The numbers in brackets are the numbers of cells required if a conventional (uniform mesh) FDTD method were to be used.

\section{RESULTS AND DISCUSSIONS}

\section{A. Experimental Validation of Calculation Method}

In order to validate the numerical simulations for large problem spaces such as the elevator modeled in this study, we carried out a simple experiment to measure the input characteristics of the dipole antenna close to a metallic wall. The significance of this experiment is that it closely imitates the situation of a user positioned close to the wall when using a mobile phone inside the elevator.

The experiment was conducted in an anechoic chamber with a metallic floor. The validation dipole antenna, Type IXD-090 S/N 0209, manufactured by IndexSAR Ltd., Newdigate, U.K. [27] with a length of $149 \mathrm{~mm}$ and a diameter of $3.8 \mathrm{~mm}$, was mounted parallel to the floor. The antenna input characteristics were recorded at several distances from the floor at intervals of $20 \mathrm{~mm}$ using an HP 8757ET network analyzer. The numerical model of the wall used to provide the simulation results for comparison is shown in Fig. 4.

The measured and calculated input impedances in the freespace were $71.81-j 24.23$ and $71.79-j 33.88 \Omega$, respectively. Fig. 5 shows the input impedance of the antenna as a function of the distance away from the metallic floor. Both simu-

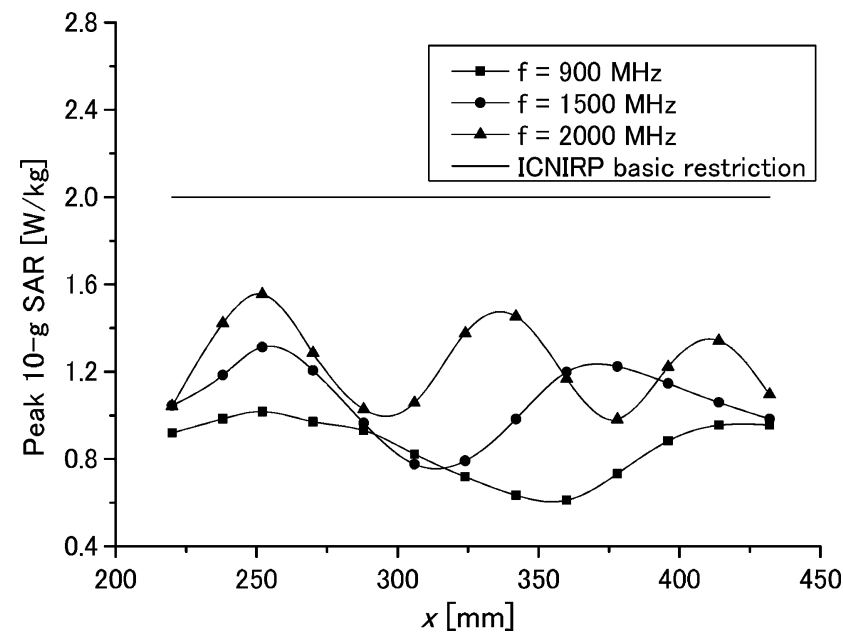

Fig. 6. Variation of the peak 10-g SAR of a passenger in the elevator as a function of the distance, $x$, from the wall, with antenna output power of $250 \mathrm{~mW}$ at 900,1500 , and $2000 \mathrm{MHz}$. The distance from the wall along the $y$-axis was fixed at $y=170 \mathrm{~mm}$.

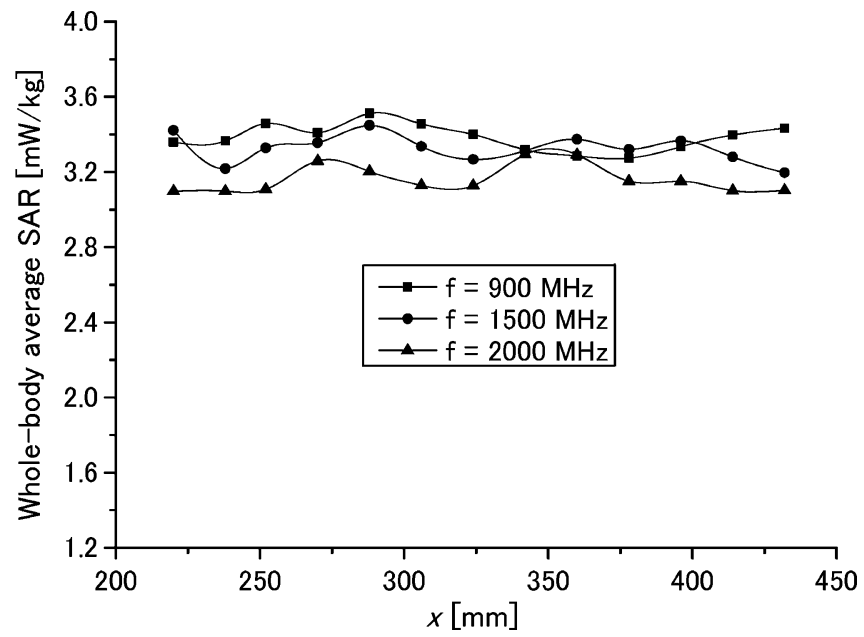

Fig. 7. Variation of the whole-body average SAR of a passenger in the elevator as a function of the distance $x$, from the wall, with antenna output power of $250 \mathrm{~mW}$ at 900,1500 , and $2000 \mathrm{MHz}$. The distance from the wall along the $y$-axis was fixed at $y=170 \mathrm{~mm}$.

lation and measured results are plotted in this figure. The input impedance of the antenna close to a metallic wall was observed to vary in a quasi-sinusoidal manner as it was moved away from the wall and its values were found to approach the free-space values at the far distance from the wall. Good agreement between the two results was obtained. The discrepancy in the reactance between the measured and calculated results observed in Fig. 5(b) can be attributed to the difficulties in completely isolating the dipole antenna and its driving transmission line during the measurements. 


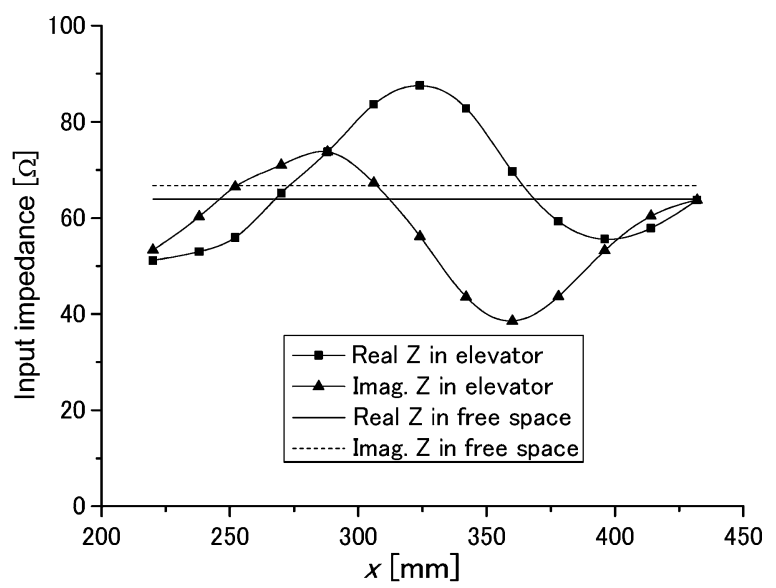

(a)

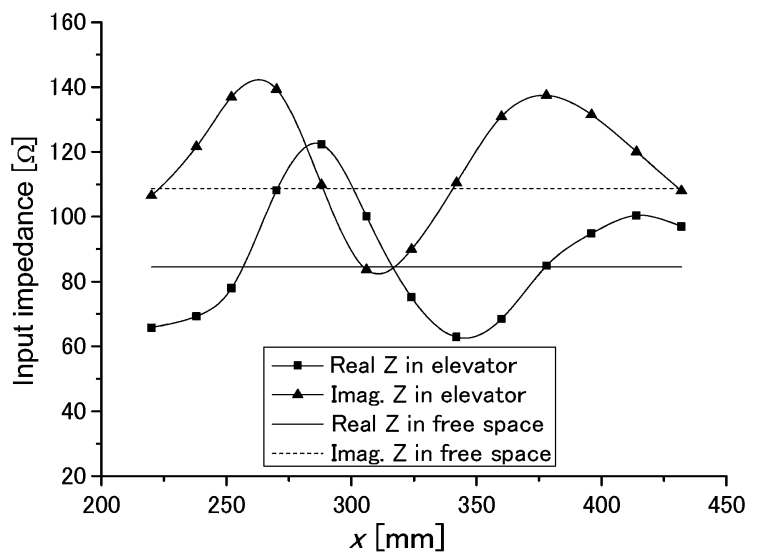

(b)

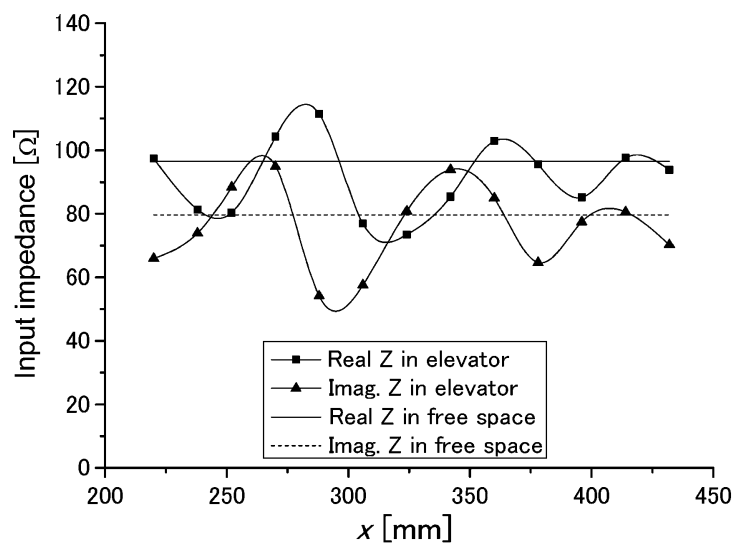

(c)

Fig. 8. Input impedance of the half-wavelength dipole antenna inside the elevator as a function of the distance $x$ from the wall. (a) $900 \mathrm{MHz}$. (b) $1500 \mathrm{MHz}$. (c) $2000 \mathrm{MHz}$. The distance from the wall along the $y$-axis was fixed at $y=$ $170 \mathrm{~mm}$.

\section{B. SAR of the Human Model in Free Space}

This section investigates the SAR of the human model in free space. This is necessary since the data obtained are needed for comparison with those values obtained in the elevator.

The problem space was assumed to be of the same size as that used when modeling the elevator, i.e., Fig. 3. Even with the use of nonuniform mesh FDTD modeling, the total memory requirement for the problem was about $52 \mathrm{~GB}$. A wall clock time of $1.3 \mathrm{~h}$ was required to complete a 15 -period simulation using a supercomputer (NEC SX-8, 8 CPU).

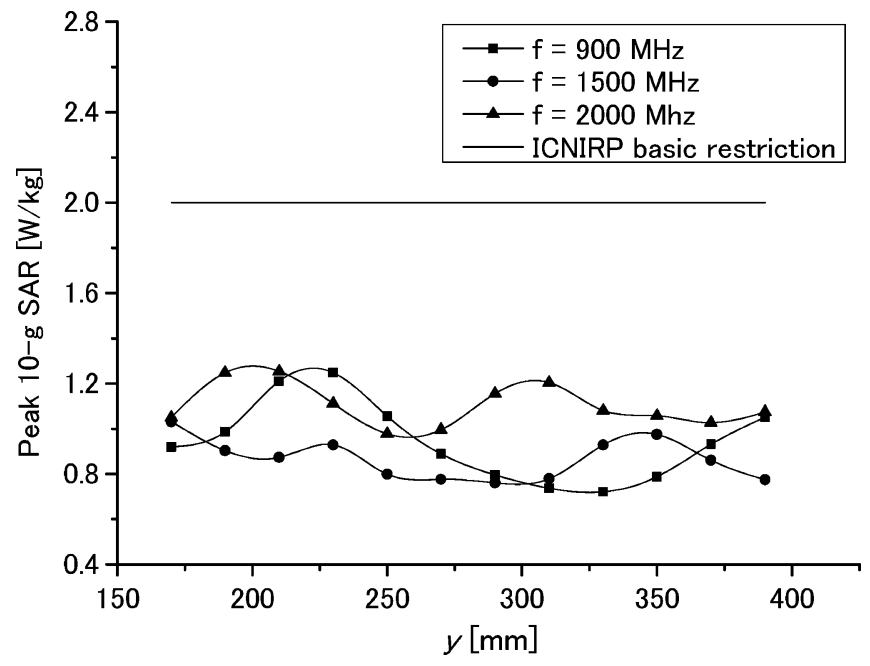

Fig. 9. Variation of the maximum 10-g SAR of a passenger in the elevator as a function of the distance $y$, from the wall, with antenna output power of $250 \mathrm{~mW}$ at 900,1500 , and $2000 \mathrm{MHz}$. The distance from the wall along the $x$-axis was fixed at $x=220 \mathrm{~mm}$.

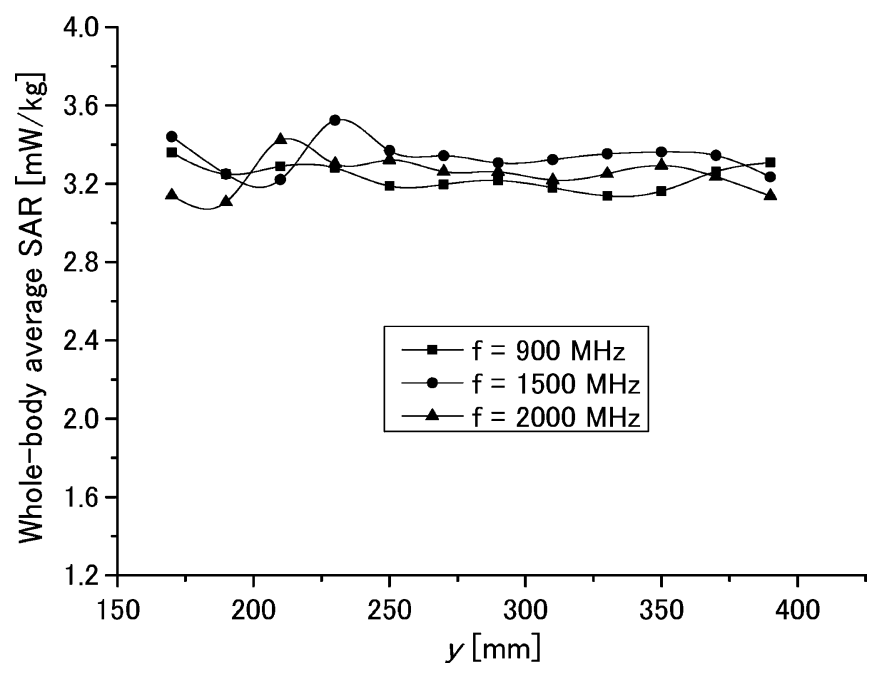

Fig. 10. Variation of the whole-body average SAR of a passenger in the elevator as a function of the distance $y$, from the wall, with antenna output power of $250 \mathrm{~mW}$ at 900,1500 , and $2000 \mathrm{MHz}$. The distance from the wall along the $y$-axis was fixed at $x=220 \mathrm{~mm}$.

A thin-wire half-wavelength dipole, here representing a mobile phone, was excited to transmit a power of $250 \mathrm{~mW}$, corresponding to the maximum power W-CDMA mobile phones used in Japan. The transmitted power $P_{t}$ is related to the real part of net input power of the antenna $P_{\text {in }}$ by the equation

$$
\begin{aligned}
P_{t} & =\operatorname{Re}\left\{\frac{1}{2}\left|V_{\text {in }}\right|^{2} / z_{\text {in }}\right\} \\
& =\frac{R}{2\left(R^{2}+X^{2}\right)}\left|V_{\text {in }}\right|^{2}
\end{aligned}
$$

where $V_{\mathrm{in}}, Z_{\mathrm{in}}, R$, and $X$ are the input voltage, impedance, reactance, and resistance, respectively. Based on the impedance values, the input voltage was set such that $P_{t}$ is always $250 \mathrm{~mW}$, representing a worst case condition. For the case of the user in free space, $P_{t}$ is given as

$$
P_{t}=P_{\mathrm{abs}}+P_{\mathrm{rad}}
$$




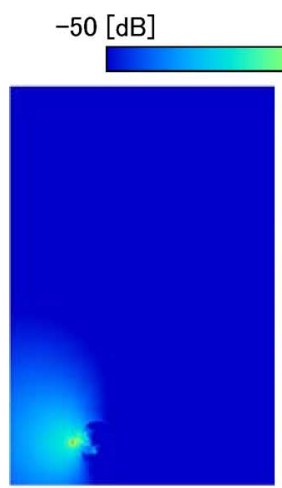

(a)

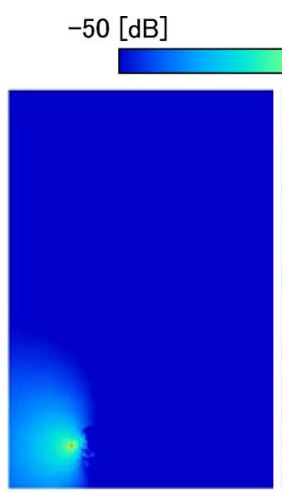

(a)

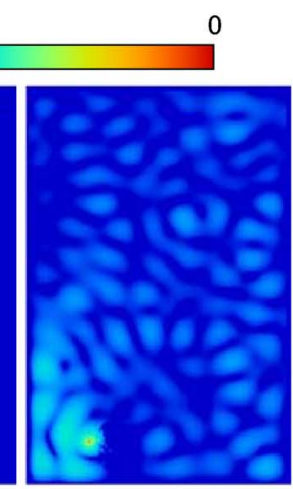

(b)

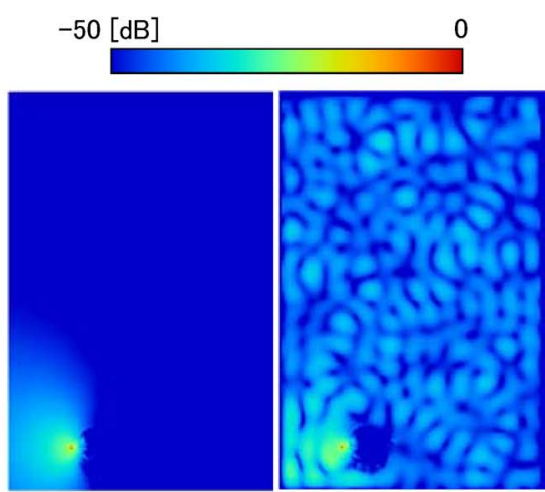

(a) (b)

(i)

(ii)

(iii)

Fig. 11. Normalized electric field distribution in the $x z$-plane. (a) Free space. (b) Elevator. (i) $900 \mathrm{MHz}$. (ii) $1500 \mathrm{MHz}$ (iii) $2000 \mathrm{MHz}$. The passenger is positioned at $x=252$ and $y=170 \mathrm{~mm}$.

where $P_{\text {abs }}$ is the power absorbed by the user and $P_{\text {rad }}$ is the power radiated in free space. When the passenger is in the elevator, the transmitted power is given as

$$
P_{t}=P_{\text {abs }}+P_{\text {hole }}
$$

where $P_{\text {hole }}$ is the power escaping through the openings at the top of the elevator. For a fully enclosed elevator, $P_{\text {hole }}$ would be equal to zero, implying that all power from the antenna is absorbed by the passenger.

Peak spatial-averaged SAR were calculated according to ICNIRP guidelines in which averaging volumes that included both the head and pinna were used in the calculation. Furthermore, the spatial average SAR was evaluated in contiguous tissues that contain a mass within $5 \%$ of $10 \mathrm{~g}$.

Table I summarizes the input impedance of the half-wavelength dipole antenna, peak 10-g SAR, whole-body average SAR, and absorbed power of the human model in free space for the antenna output power of $250 \mathrm{~mW}$.

The spatial 10 -g SAR values were $45 \%, 47 \%$, and $57 \%$ of the ICNIRP basic restriction; i.e., $2 \mathrm{~W} / \mathrm{kg}$ at 900,1500 , and $2000 \mathrm{MHz}$, respectively. The whole-body average SAR values were found to be $2.6 \%, 1.8 \%$, and $1.4 \%$ of the basic restriction; i.e., $0.08 \mathrm{~W} / \mathrm{kg}$ at 900,1500 , and $2000 \mathrm{MHz}$, respectively. Siegbahn et al. [14] have calculated the whole-body average SAR of the simplified homogeneous model of the visible human model using the FDTD method at $900 \mathrm{MHz}$. Under antennatransmitted power similar to that used in this study, the wholebody average SAR obtained was $2.3 \%$ of the ICNIRP basic restriction.

\section{SAR of the Human Model Inside the Elevator}

In this section, we investigate the SAR dependence on the passenger position inside the elevator using the nonuniform FDTD modeling of Fig. 3. It should be noted that if the conventional FDTD method were to be used, this problem would require about $248 \mathrm{~GB}$ of memory, almost five times that needed for a nonuniform mesh FDTD analysis. More than 200 periods were required to simulate this problem depending on the frequency of operation and user position in the elevator. A wall clock time of $5.3 \mathrm{~h}$ was used to complete the simulation for 200 periods at $900 \mathrm{MHz}$ using a supercomputer (NEC SX-8, 8 CPU).

1) SAR in the $x$-Direction: The position of the passenger in the elevator is described in terms of $x$ and $y$, as indicated in Fig. 3.

Fig. 6 shows the peak 10-g SAR of the passenger in the elevator at an antenna output power of $250 \mathrm{~mW}$. The values are plotted as a function of the position from the wall in the $x$-direction. The distance from the wall in the $y$-direction is kept constant at $y=170 \mathrm{~mm}$. The distances $x=220 \mathrm{~mm}$ and $y=170 \mathrm{~mm}$ are the minimum valid distances in the $x$ - and $y$-directions in which the body of the passenger is already touching the walls in those directions. The ICNIRP 10-g SAR limit for general public exposure is also indicated in the graph.

We observed from the graph that the peak 10-g SAR varies in a slow decaying quasi-sinusoidal wave with $x$. Furthermore, we found that the peaks of the sine wave occurred at the distance $x_{\text {peak }}$ from the wall given approximately by the equation

$$
x_{\text {peak }}=(2 n+1) \frac{\lambda}{4}
$$

where $n=0,1,2, \ldots$ and $\lambda$ is the free-space wavelength at a given frequency. The maximum values of the peak 10-g SAR in the graphs were obtained at a distance of about $252 \mathrm{~mm}$ from the wall, which corresponds to $n=3,5$, and 7 at 900 , 1500 and $2000 \mathrm{MHz}$, respectively. The maximum 10-g SAR values obtained at 900, 1500, and $2000 \mathrm{MHz}$ were $1.02,1.30$, and $1.56 \mathrm{~W} / \mathrm{kg}$, respectively, corresponding to an increase of $13.2 \%, 40.1 \%$, and $37.6 \%$ over those of the human model in free space. Furthermore, these values were 51\%, 66\%, and 78\% of the ICNIRP's peak 10-g SAR basic restrictions.

Another interesting fact is that the destructive interference of the EMFs due to multireflections in the enclosed environments was so strong that sometimes the peak 10-g SAR of the passenger in the elevator was lower than that of the user in free space.

The whole-body average SAR as a function of the distance in the $x$-direction is given in Fig. 7. We observe that the curves for 


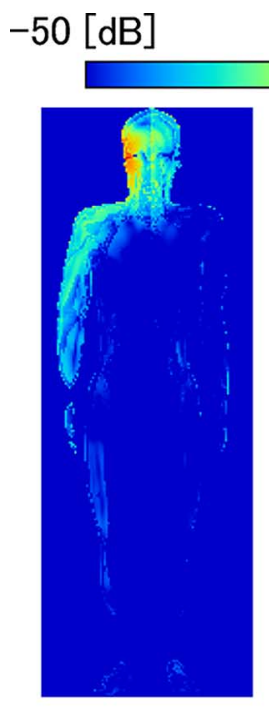

(a)

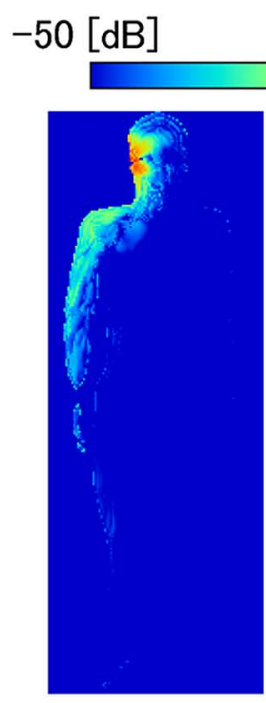

(a)

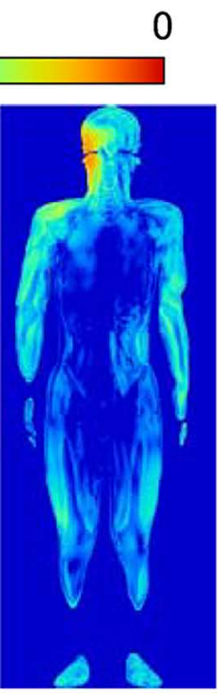

(b)

(i)

(a)

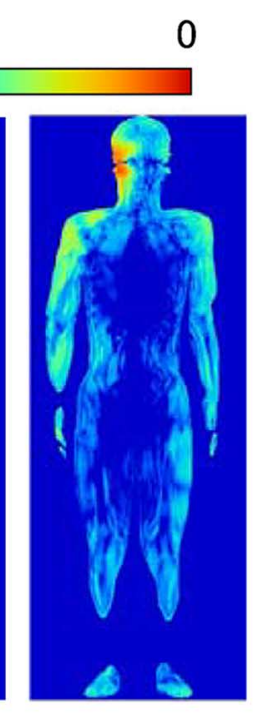

(b)

(ii)

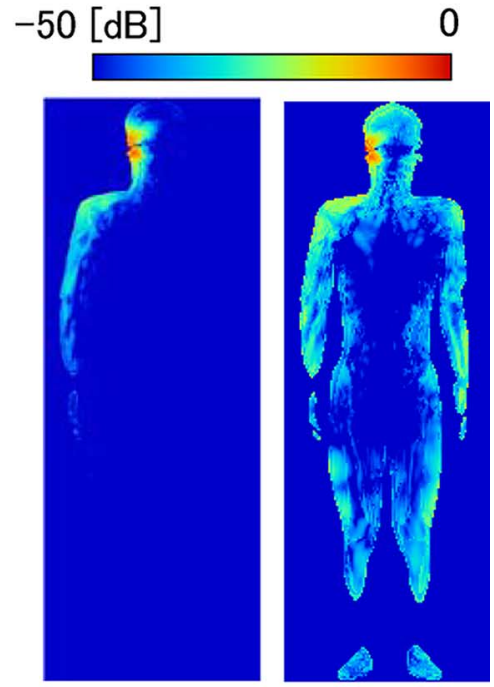

(a) (b)

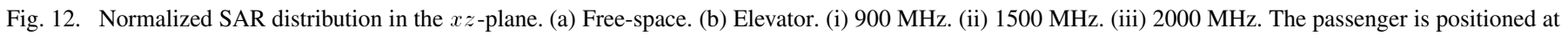
$x=252$ and $y=170 \mathrm{~mm}$.

the whole-body average SAR do not follow a sinusoidal pattern like those of the peak 10-g SAR. The reason for this may be that the whole-body average SAR depends on the amount of power absorbed by the passenger, which, in turn, depends on the amount of power escaping through the holes at the top of elevator.

Unlike the peak 10-g SAR, all of the whole-body average SARs in the elevators were larger than the free-space values. The maximum values of the whole-body average SAR were larger than those of the user in free space by $71 \%, 135 \%$, and $188 \%$, respectively, at 900, 1500, and $2000 \mathrm{MHz}$. However, these values were only $4.4 \%, 4.3 \%$, and $4.1 \%$ of the ICNIRP whole-body exposure limit of $0.08 \mathrm{~W} / \mathrm{kg}$. Even in the case of a completely covered elevator, in which all power transmitted by the antenna were absorbed by the passenger, the whole-body average SAR obtained was $3.61 \mathrm{~mW} / \mathrm{kg}$, which is only $4.5 \%$ of the limit. We can conclude that for the single mobile phone user inside the elevator, the whole-body average SAR would not exceed the exposure limit recommended by the international guidelines.

The input impedance of the dipole antenna in the elevator as a function of $x$ is plotted in Fig. 8. The results for the human model in free space are also indicated in the graph. The antenna input impedance was seen to vary with $x$, indicating a strong coupling between the antenna and walls. Like the peak $10-\mathrm{g}$ SAR, the resistance and reactance were also seen to vary in the sinusoidal form, implying that the matching of the antenna is also a function of the position of the user in the elevator.

2) SAR in the $y$-Direction: We have also investigated the variation of the SAR along the $y$-axis. In this case, the distance from the wall in the $x$-direction was fixed at $x=220 \mathrm{~mm}$, which is the closest position to the wall in the $x$-direction. The result of the peak 10-g SAR is shown in Fig. 9. They were observed to vary in a clear sinusoidal form, especially for the 900-

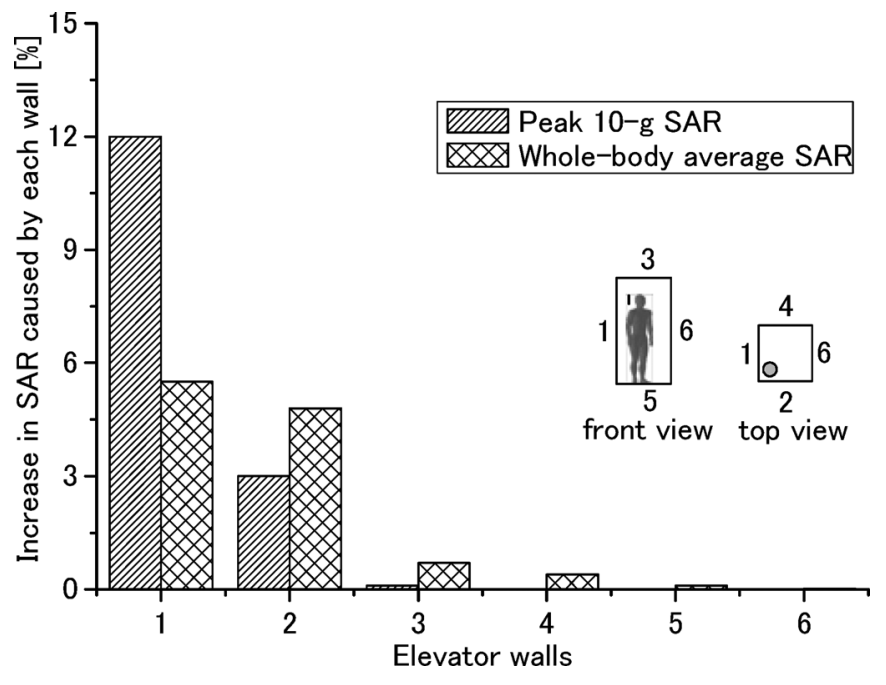

Fig. 13. Effect of the reflections from each wall of the elevator on the SAR when the passenger is placed in a position of maximum SAR in the elevator at $900 \mathrm{MHz}$.

and 2000-MHz curves. The peak 10-g SAR were obtained at $x=230,170$, and $210 \mathrm{~mm}$, respectively, at 900, 1500, and $2000 \mathrm{MHz}$. The effect of varying the passenger position in the $y$-direction is found to have very little effect on the whole-body average SAR, as seen in Fig. 10. The input characteristics (not shown) of the antenna were also found to vary with position in the $y$-axis.

\section{Electric Field and SAR Distribution}

Fig. 11 shows the typical electric field distributions at 900, 1500 , and $2000 \mathrm{MHz}$ for the passenger in free space and in an elevator. The passenger in the elevator was placed at a position for maximum 10 -g SAR, varying in the $x$-direction; i.e., at $x=$ 
TABLE II

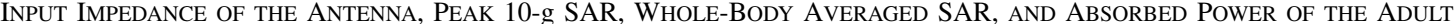
Female Model When Half-WAVElength Dipole ANTENNA IS TRANSMitTing 250 mW AT 900, 1500, AND 2000 MHz

\begin{tabular}{ccccc}
\hline \hline Frequency [MHz] & $\begin{array}{c}\text { Input Impedance } \\
{[\Omega]}\end{array}$ & $\begin{array}{c}\text { Peak 10-g SAR } \\
{[\mathrm{W} / \mathrm{kg}]}\end{array}$ & $\begin{array}{c}\text { Whole-body averaged } \\
\text { SAR }[\mathrm{mW} / \mathrm{kg}]\end{array}$ & $\begin{array}{c}\text { Absorbed } \\
\text { Power }[\mathrm{mW}]\end{array}$ \\
\hline 900 & $65+\mathrm{j} 65$ & 0.91 & 2.68 & 144 \\
1500 & $78+\mathrm{j} 102$ & 0.96 & 2.14 & 115 \\
2000 & $85+\mathrm{j} 78$ & 1.09 & 1.71 & 92 \\
\hline \hline
\end{tabular}

$252 \mathrm{~mm}, y=170 \mathrm{~mm}$. To see the difference between the two cases more clearly, the problem space used for the passenger in the elevator was also used for the free-space calculation. The fields are observed in the $x y$-plane cut at the antenna feed point. The standing waves due to multireflection from elevator walls are observed in Fig. 11(i)(b), 11(ii)(b), and 11(iii)(b). Standings waves depends on the antenna operating frequency with more standing waves formed at $2000 \mathrm{MHz}$. The maximum electric field values for both the elevator and free space were obtained near the antenna feed point.

The corresponding SAR distributions in the $x z$-plane of the human model in free space and in the elevator are given in Fig. 12. The difference between SAR distribution in free space and the elevator can be easily observed in these figures. The dependency of SAR distribution on the frequency is more visible in the free-space case that in the elevator. This may be the reason for the small difference in the whole-body SAR obtained at 900, 1500 , and $2000 \mathrm{MHz}$ for the elevator case. Although we performed several numerical calculations of this model, covering a distance of up to $414 \mathrm{~mm}$ and $390 \mathrm{~mm}$ in the $x$ - and $y$-directions, respectively, we did not find any unusual SAR that could be attributed to phenomena such as hot spots [3]. All maximum SAR values obtained occurred in the head area near the antenna, as depicted in the SAR distribution of Fig. 12.

\section{E. Effects of Elevator Structures}

In the above sections, we have shown that peak 10-g SAR depends on the passenger position relative to the elevator walls, and the most influential factor for whole-body average SAR was the amount of power escaping through the holes at the top of the elevator. In this section, we investigate the effects of the reflections of EMFs from each wall of the elevator. We assume that the passenger is in position for maximum peak 10-g SAR, i.e., at position where $x=252 \mathrm{~mm}$ and $y=170 \mathrm{~mm}$ in the elevator. We consider only the frequency of $900 \mathrm{MHz}$. With the passenger in this position, one wall of the elevator is left in its position, while the remaining five walls are removed from the simulation. The SAR values were found to increase over the free-space values, as shown in Fig. 13. Wall number 1, i.e., the wall parallel and closest side of the head with the phone, was found to have greater effect than that from the other walls. It can be observed from Fig. 13 that the top wall had an insignificant effect to both peak 10-g and whole-body average SAR.

Interestingly, when we placed the passenger in the position for the minimum SAR, i.e., where $x=396 \mathrm{~mm}$ and $y=$ $170 \mathrm{~mm}$, the values of the SAR (not shown) were found to decrease when compared with the free-space value. Again, the effect was more pronounced for wall number 1 followed by wall number 2. This was just like the case shown in Fig. 13: the top wall had a very small effect. The reason for the increase and decrease in SAR is the constructive and destructive interference between the incident fields and the reflected fields from the walls. It should be noted, however, that the role of walls 1 and 6 and walls 2 and 4 are interchangeable if the phone was placed on the other side of the head and the passenger was placed at the back of the elevator.

Generally, we can conclude that the increased SAR in the enclosed environment is a result of direct reflections of the EMFs from the walls close to the side of the head of the passenger with the phone. As shown in this paper, their positions can be predicted numerically. Furthermore, the introduction of the holes at the top of the elevator used in our model did not affect the conservativeness of the results obtained in this study. It should be noted that most previous studies on this subject employed a completely enclosed as one of the worst case conditions.

\section{F. Comparison With Other Human Models}

In order to find out if the results obtained in the above sections are applicable to other human models, we compared the obtained results with those of the realistic Japanese adult female model.

Table II lists the dipole input impedance, SAR, and absorbed power of the female model for different frequencies when the model was in free space. When compared with the results of the adult male shown in Table I, an insignificant difference was observed in the input characteristics of the antenna. The difference in peak 10-g SAR was less than 4\%. However, the whole-body average SAR of the female model exhibited higher values due to its lighter weight. As a result, differences were recorded between the male and female of nearly $30 \%, 47 \%$, and $51 \%$ at 900 , 1500 , and $2000 \mathrm{MHz}$, respectively.

To compare the effects of multireflection between the models, we also calculated the SAR of the adult female model as a function of distance from the walls. We positioned the model exactly at the same positions used for the male model. Fig. 14 shows the 10-g and whole-body average SAR of the male and female. From the figure, we can see that the curves for peak 10-g SAR followed similar patterns. This finding is very important since it implies that the characteristics of the peak 10-g SAR of a human model in enclosed environments are the same regardless of the type of model used.

The higher values of whole-body average SAR of the female model were due to its low weight compared to the male model's weight. The maximum value obtained for the female model was only $5 \%$ of the ICNIRP's whole-body average SAR basic restriction. 


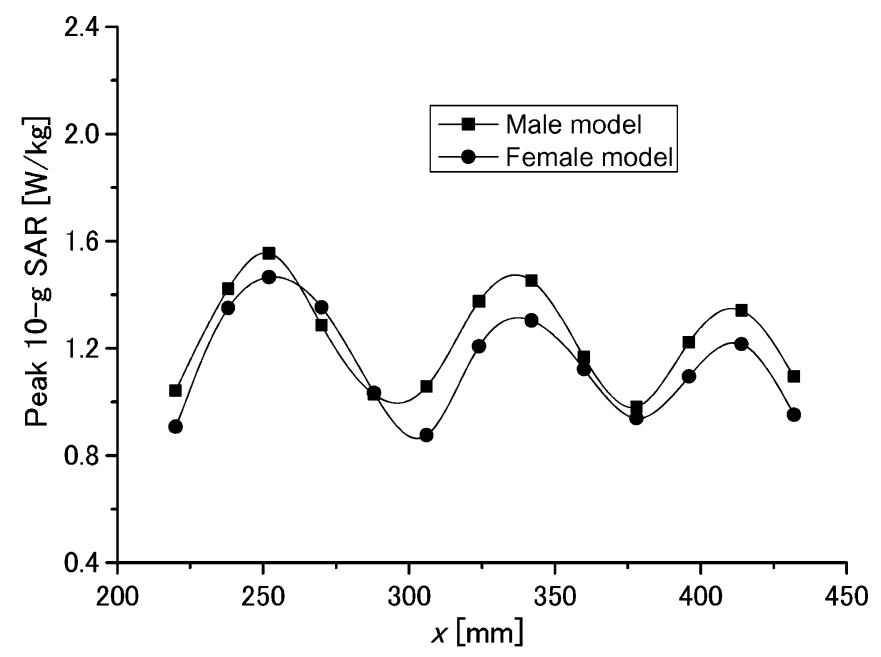

(a)

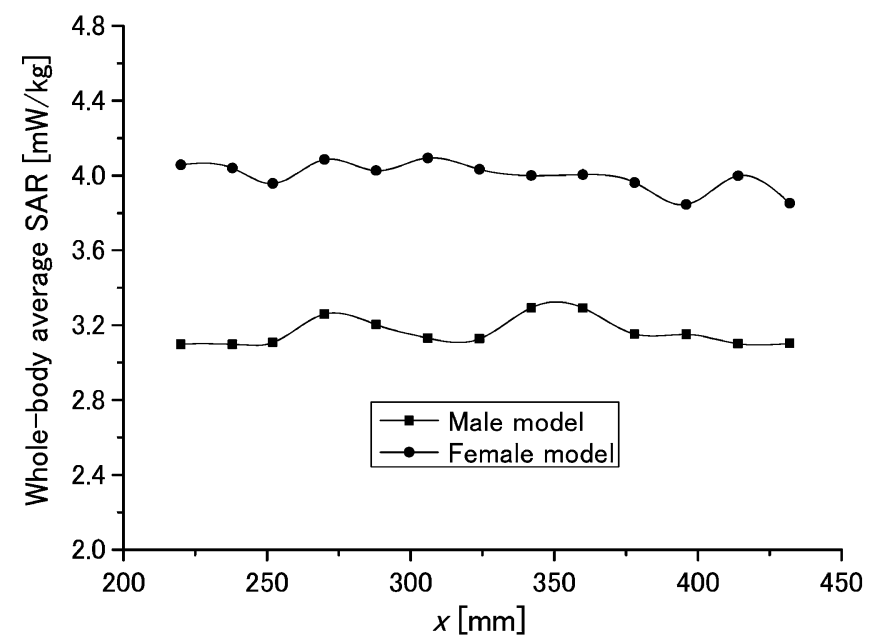

(b)

Fig. 14. Variation of the SAR of the adult male and female model in the elevator as a function of the distance $x$ from the wall for the antenna output power of $250 \mathrm{~mW}$ at $2000 \mathrm{MHz}$. (a) Maximum 10-g SAR. (b) Whole-body average SAR.

\section{CONCLUSION}

This paper has presented a detailed investigation of the SAR in anatomically high-resolution Japanese models in the elevator at the three mobile phone frequency bands used in Japan. A worst case condition that includes: 1) a half-wavelength dipole antenna placed near the head without hand [23]; 2) a vertically oriented dipole antenna [24], [25] parallel to the sidewalls of the elevator for maximum reflection; 3) an elevator with metallic walls; and 4) constant maximum transmitted power of $250 \mathrm{~mW}$ was considered.

The SARs were found to depend on the passenger's position in the elevator. The results obtained indicate that the whole-body average SAR of a single mobile phone user inside an elevator will not exceed the basic restrictions recommended in the ICNIRP and IEEE guidelines. Although the values of the peak 10-g SAR presented in this study did not exceed the exposure limit, a substantial enhancement due to multireflections from the elevator walls was observed. These finding are true regardless of the type of the human model used.

Furthermore, the results obtained in this study should be valid for other types of enclosed environments such as trains and cars with an even smaller effect than that observed in an elevator. This is because cars and trains contain absorbing materials such as seats and nonmetal parts in the walls such as glass windows. This would result in less reflection from the walls.

The other concern of the work presented here, besides that of finding whether or not the exposure guide lines are exceeded in elevators, was to provide an understanding of the SAR characteristics of the mobile phone user in such environments, under a worst case condition. We hope that this study will contribute to a better understanding, especially in the peak spatial-averaged SAR in these environments.

\section{ACKNOWLEDGMENT}

Parts of the calculations in this study have been conducted using the supercomputer (NEC/SX-8R) at the National Institute of Information and Communications Technology, Tokyo, Japan.

\section{REFERENCES}

[1] ICNIRP, "Guidelines for limiting exposure to time-varying electric, magnetic and electromagnetic fields (up to $300 \mathrm{GHz}$ )," Health Phys. Soc., vol. 74, no. 4, pp. 494-522, Apr. 1998.

[2] IEEE Standards for Safety Levels with Respect to Exposure to Radio Frequency Electromagnetic Fields, $3 \mathrm{kHz}$ to $300 \mathrm{GHz}$, IEEE Standard C95.1-2005, 2005.

[3] T. Hondou, "Rising level of public exposure to mobile phones: Accumulation through additivity and reflectivity," J. Phys. Soc. Jpn., vol. 71, no. 2, pp. 432-435, Feb. 2002.

[4] T.Hondou, "Physical validity of assumptions for public exposure to mobile phones," J. Phys. Soc. Jpn., vol. 71, no. 12, pp. 3101-3102, Dec. 2002.

[5] T. Hondou, T. Ueda, Y. Sakata, N. Tanigawa, T. Suzuki, T. Kobayashi, and K. Ikeda, "Passive exposure to mobile phones: Enhancement of intensity by reflections," J. Phys. Soc. Jpn., vol. 75, no. 8, pp. 0848011-08481-5, Aug. 2006.

[6] A. Kramer, J. Frohlich, and N. Kuster, "Towards danger of mobile phones in planes, trains, cars, and elevators," J. Phys. Soc. Jpn., vol. 71, no. 12, pp. 3100-3100, Dec. 2002.

[7] C. H. Chou, “'Trapped' radiation,” New Sci. Mag. vol. 174, no. 2348, Jun. 2002. [Online]. Available: http://space.newscientist.com/article/ mg17423486.000-trapped-radiation.html

[8] A. Tropainen, "Human exposure by mobile phones in enclosed areas," Bioelectromagnetics, vol. 24, no. 1, pp. 63-65, Jan. 2003.

[9] T. Hikage, T. Nojima, S. Watanabe, and T. Shinozuka, "Electric-field distribution estimation in a train carriage due to cellular radios in order to assess the implantable cardiac pacemaker EMI in semi-echoic environments," IEICE Trans. Commun., vol. E88-B, no. 8, pp. 3281-3286, Aug. 2005.

[10] J. Ferrer et al., "On the exposure to mobile phone radiation in trains," Appl. Phys. Lett., vol. 86, pp. 224 101-1-224 101-3, 2005.

[11] M. Martinez-Burdalo, L. Nonidez, A. Martin, and R. Villar, "FDTD analysis of the maximum SAR when operating a mobile phone near a human eye and a wall," J. Phys. Soc. Jpn., vol. 71, no. 12, pp. 3101-3102, Dec. 2002.

[12] J. Cooper and V. Hombach, "The specific absorption rate in a spherical head model from a dipole with metallic walls nearby," IEEE Trans. Microw. Theory Tech., vol. 40, no. 4, pp. 377-382, Nov. 1998.

[13] H. Dominguez, A. Raizer, and W. P. Carpes, Jr., "Electromagnetic fields Radiated by a cellular phone in close proximity to metallic walls," Magnetics, vol. 38, no. 2, pp. 793-796, Mar. 2002.

[14] M. Siegbahn, H. Gradin, B. Thors, and C. Tornevik, "RF exposure from multiple mobile phones in an enclosed environment," in Abstracts Bioelectromagn. Soc. Annual Meeting, Jun. 2006, pp. 499-502.

[15] A. Y. Simba, T. Hikage, S. Watanabe, and T. Nojima, "The dependence of SAR upon position of a mobile phone user in enclosed environments," in 29th Bioelectromagn. Annu. Abstract Collection Meeting, Jun. 2007, pp. 256-258.

[16] H. Jiang and H. Arai, "Analysis of computational error in antenna's simulation by using non-uniform mesh FDTD," IEICE Trans. Commun., vol. E83-B, no. 7, pp. 1544-1553, Jul. 2000.

[17] H. Meskanen and O. Pekonnen, "FDTD analysis of field distribution in an elevator car by using various antenna positions and orientations," Electron. Lett., vol. 34, no. 6, pp. 534-535, Mar. 1998. 
[18] T. Nagaoka, S. Watanabe, K. Sakurai, E. Kunieda, S. Watanabe, M. Taki, and Y. Yamanaka, "Development of realistic high-resolution whole-body voxel models of Japanese adult males and females of average height and weight, and application of the models to radio-frequency electromagnetic-field dosimetry," Phys. Med. Biol., vol. 4, pp. 2319-2329, Dec. 2003

[19] C. Gabriel, "Compilation of the dielectric properties of body tissues at RF and microwave frequencies," Brooks Air Force Base, San Antonio, TX, Tech. Rep. AL/OE-TR-1996-0037, 1996.

[20] C. Gabriel, S. Gabriel, and E. Corhout, "1996a: The dielectric properties of biological tissues: I. Literature surveys," Phys. Med. Biol., vol. 41, pp. 2231-2249, Dec. 1996.

[21] S. Gabriel, R. W. Lau, and C. Gabriel, "1996b: The dielectric properties of biological tissues: II. Measurements in frequency range $10 \mathrm{~Hz}$ to 20 GHz," Phys. Med. Biol., vol. 41, pp. 2251-2269, Dec. 1996.

[22] S. Gabriel, R. W. Lau, and C. Gabriel, "1996c: The dielectric properties of biological tissues: III. Parametric models for the dielectric spectrum of tissues," Phys. Med. Biol., vol. 41, pp. 2271-2293, Dec. 1996.

[23] M. Okoniewsky and A. Stuchly, "A study of the handset antenna and human body interaction," IEEE Trans. Microw. Theory Tech., vol. 47, no. 8, pp. 1528-1534, Aug. 1999.

[24] P. J. Dimbylow and S. M. Mann, "SAR calculations in an anatomically realistic model of the head for mobile communication transceivers at $900 \mathrm{MHz}$ and $1.8 \mathrm{GHz}, "$ Phys. Med. Biol., vol. 39, pp. 1537-1553, 1994.

[25] A. D. Tinniswood, C. M. Furse, and O. P. Gandhi, "Computations of SAR distributions for two anatomically based models of the human head using CAD files of commercial telephones and the parallelized FDTD code," IEEE Trans. Antennas Propag., vol. 46, no. 6, pp. 829-833, Jun. 1998.

[26] J. P. Berenger, "A perfectly matched layer for the absorptions of electromagnetic waves," J. Comput. Phys., vol. 114, no. 2, pp. 185-200, Oct. 1994.

[27] “Mapsar2 System Manual" IndexSAR Ltd., Newdigate, U.K., 2005. [Online]. Available: http://www.indexsar.com/, (operating instructions for hardware and software)

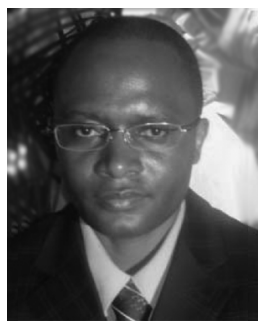

Ally Y. Simba (S'01-M'06) was born in Bagamoyo, Tanzania. He received the B.Sc. degree (with honors) in electronics science and communication from the University of Dar es salaam, Dar es Salaam, Tanzania, in 1998, and the M.E and Ph.D. degrees in electronics and information engineering from Hokkaido University, Sapporo, Japan, in 2003 and 2006.

In 2006, he joined the Biomedical Electromagnetic Compatibility (EMC) Group, National Institute of Information and Communications Technology, Tokyo, Japan, as an Expert Researcher. His recent research interest is bioelectromagnetics.

Dr. Simba is a member of the Institute of Electronics, Information and Communication Engineers (IEICE), Japan, and the Institute of Engineering and Technology (IET), U.K. He was the recipient of the Japanese Government Scholarship (Monbusho Scholarship) (2000-2006).

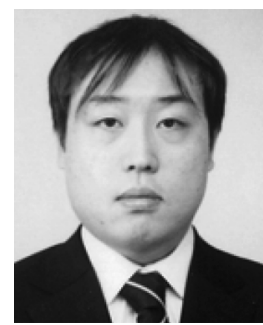

$(\mathrm{EMC})$ issues.

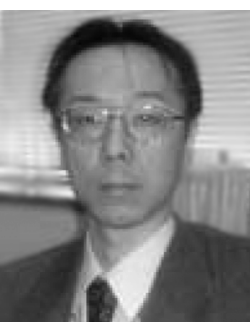

Soichi Watanabe (S'93-M'96) received the B.E., M.E., and D.E., degrees in electrical engineering from Tokyo Metropolitan University, Tokyo, Japan, in 1991, 1993, and 1996, respectively.

$\mathrm{He}$ is currently with the National Institute of Information and communications Technology (NICT), Tokyo, Japan. His main research interest is biomedical electromagnetic compatibility.

Dr. Watanabe is a member of the Institute of Electronics, Information and Communication Engineers (IEICE), Japan, the Institute of Electrical Engineers (IEE), Japan, and the Bioelectromagnetics Society. Since 2004, he has also been a member of the Standing Committee on Physics and Engineering of the ICNIRP. He was the recipient of several awards, including the 1996 International Scientific Radio Union (URSI) Young Scientist Award and the 1997 IEICE Best Paper Award

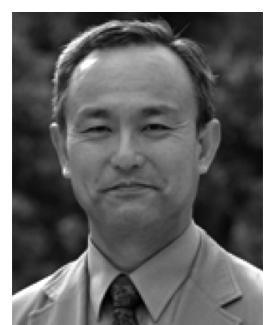

Toshio Nojima (S'73-M'74) received the B.E. degree in electrical engineering from Saitama University, Saitama, Japan, in 1972, and the M.E. and $\mathrm{Ph} . \mathrm{D}$. degrees in electronic engineering from Hokkaido University, Sapporo, Japan, in 1974 and 1988, respectively.

From 1974 to 1992, he was with Nippon Telegraph and Telephone (NTT) Communications Laboratories, where he was engaged in the development of high-capacity microwave long-line relay systems (SSB-AM and 256QAM systems). From 1992 to 2001, he was with NTT DoCoMo Inc., where he was a Senior Executive Research Engineer and conducted research on the radio safety issues related to mobile radio systems, as well as high-efficiency radio equipment technologies. Since January 2002, he has been a Professor with the Graduate School of Information Science and Technology, Hokkaido University.

Dr. Nojima is a member of the Bioelectromagnetics Society (BEMS), the Institute of Electrical Engineers (IEE), Japan, and the Institute of Electronics, Information and Communication Engineers (IEICE), Japan. 